\title{
The shear viscosity of carbon fibre suspension and its application for fibre length measurement
}

\author{
Guozhan Jiang, ${ }^{1}$ Thomas A Turner, Stephen J Pickering
}

Division of Materials, Mechanics and Structures,

University of Nottingham, Nottingham NG7 2RD, United Kingdom

\begin{abstract}
The viscosity of short carbon fibre suspensions in glycerol aqueous solution was measured using a bespoke vane-in-cup viscometer, where the carbon fibre has an aspect ratio from 450 to 2209 . In the semi-concentrated regime, $\mathrm{nL}^{3}$ ranging from 20 to 4400 , the suspensions demonstrated strong shear-thinning characteristics particularly at higher concentrations. The shear-thinning characteristic is strongly related to the crowding factor proposed by Kerekes, indicating that non-hydrodynamic interactions occur in the suspensions. The influence of fibre bending on viscosity emerges when the bending ratio is lower than 0.0028 . An empirical model based on transient network formation and rupture was proposed and used to correlate the relative viscosity with fibre concentration $\mathrm{nL}^{3}$ and shear rate. Based on the model, a viscosity method is established to analyse the fibre length by measuring the viscosity of the fibre suspension using a bespoke vane-in-cup viscometer.
\end{abstract}

\section{Key words}

Carbon fibre, suspension, viscosity, vane-in-cup, shear-thinning

\footnotetext{
${ }^{1}$ Current address: School of Biology, Chemistry and Forensic Science, University of Wolverhampton, Wolverhampton WV1 1LY, UK; Email: Guozhan.jiang@wlv.ac.uk
} 


\section{Introduction}

Recycling high value carbon fibres from the waste streams of carbon fibre reinforced plastics has been extensively investigated for nearly two decades, and is now being commercially operated. Notwithstanding the recycling processes, the recycled carbon fibres are usually in a fluffy short form, with a length of about 3-12 $\mathrm{mm}$ and retaining about $70-100 \%$ of the original fibres' strength (Jiang et al. 2009; Meyer et al. 2009; Yip et al. 2002). Previous research mainly focuses on the development of recycling processes and how to reuse these recycled fibres. For the purposes of quality control, there still lacks an efficient method to assess the properties of the recycled fibres. The widely used image analysis for fibre length measurement and the single fibre tensile testing for fibre strength measurement, both require measuring over one hundred single filaments, selected from a sample containing millions of such filaments, which is highly insufficient. To provide online or offline quality control for a recycling process, these methods are not only extremely time-consuming but also suffer subjectivity during the selection. A fast and unbiased analysis method is therefore desirable.

The addition of fibres to a viscous liquid will result in an increased liquid viscosity. The magnitude of the increase is dependent on the concentration, geometry and orientation distribution of the dispersed fibres (Petrie 1999). A faster analysis method for fibre length would be made possible by measuring the fibre suspension's viscosity. Furthermore, a faster analysis method for fibre strength would also be possible based on viscosity measurement. Because at a high shear rate, the fibres will be broken down, the reduction in the average length of the fibres will result in the reduction of the fibre suspension viscosity, and the extent of this reduction in viscosity would be related to the strength of the initial fibres. Due to the simplicity in generating shear flow, the developed methods will be based on the measurement of shear viscosity. A well-defined relation between this shear viscosity and fibre length will then be integral to the methods practised.

The influence of suspended fibres upon the viscosity of a liquid receives much attention in existing literature, and the case for straight rigid fibres in dilute and semi-concentrated regime is well understood in current scholarship. In the case of a simple shear flow, the relative viscosity of fibre suspension is given in Eq. (1) (Petrich et al. 2000; Stover et al. 1992).

$$
\frac{\mu_{f}}{\mu_{s}}=1+\frac{\mu_{\text {fibre }}}{\mu_{s}}\left\langle p_{x}^{2} p_{y}^{2}\right\rangle
$$

where $p_{\mathrm{x}}$ is the component of flow direction, $p_{\mathrm{y}}$ is the component of gradient direction, $\mu_{\mathrm{f}}$ is the effective viscosity of fibre suspension, $\mu_{\mathrm{s}}$ is the viscosity of the suspending liquid, $\mu_{\text {fibre }}$ is the 
additional viscosity caused by the suspended fibres, and the item within the angle brackets is an average over the orientation distribution of the fibres.

Consider a suspension of $n$ rigid, cylindrical fibres per unit volume, and each fibre has length $L$ and diameter $d$. In the dilute regime $\left(n L^{3}<1\right)$, there are no fibre-fibre interactions, i.e. hydrodynamically independent. Batchelor (1970) derived an expression for $\mu_{\text {fibre }}$ as shown in Eq. (2);

$$
\mu_{\text {fibre }}=\frac{1}{6} \pi n L^{3} \mu_{s} \varepsilon f(\varepsilon)
$$

where $\varepsilon=[\ln (2 \mathrm{~L} / \mathrm{d})]^{-1}$, and $f(\varepsilon)=(1+0.64 \varepsilon) /(1-1.5 \varepsilon)+1.659 \varepsilon^{2}$ is a correction for the effects of finite aspect ratio. In the semi-concentrated regime $\left(n L^{3}<<(L / d)^{2}\right.$, or $\left.n L^{2} d<<1\right)$, when there are only hydrodynamic interactions between fibres, Shaqfeh and Fredrickson (1990) derived an expression for $\mu_{\text {fibre }}$ as shown in Eq. (3),

$$
\mu_{\text {fibre }}=\frac{\pi n L^{3} \mu_{s}}{3(\ln (1 / \varnothing)+\ln (\ln (1 / \varnothing)+A)}
$$

where $\phi\left(=1 / 4 n \pi d^{2} L\right)$ is the volume fraction of fibres, and $A$ is a constant related to the orientation distribution. For a suspension in which all orientations are equally probable, $A=-0.6634$. For all of the fibres which are aligned in a common direction, $A=0.1585$ (Mackaplow and Shaqfeh 1996)The orientation of fibres in a liquid flow depends on the motion of such particles. Subsequent to Jeffery's seminal work (1922) on the motion of ellipsoidal particles immersed in a viscous fluid, both experimental and further theoretical analysis on fibre orientation distribution in a shear field have been conducted. A single ellipsoidal particle suspended in a Newtonian fluid in a Stokes flow field will rotate around the vorticity axis, spending most of the time nearly aligned in the shear direction (Forgacs and Mason 1959b; Stover et al. 1992). In the semi-concentrated regime, Folgar and Tuck (1984) and Koch (1995) developed models to calculate the orientation distribution $\left\langle p_{x}{ }^{2} p_{y}{ }^{2}\right\rangle$. The evolution of fibre orientation often results in a stress overshoot and oscillations at the start-up of a shear flow measurement from an initial random orientation. After a short period of time, a steady state can be reached when an equilibrium is achieved (Dinh and Armstrong 1984), i.e. the viscosity of fibre suspension is shear-independent when there are only hydrodynamic interactions.

The above theoretical predictions work well for fibres with very short aspect ratio $(L / d)$ in dilute and semi-concentrated regime at a relatively low $\mathrm{nL}^{3}$ range. However, the majority of experimental reports (Chaouche and Koch 2001; Goto and Nagazono 1986; Kitano and Kataoka 1981; Mongruel and Cloitre 1999; Rosinger et al. 1974) indicate that fibre suspension in a semi-concentrated regime is shear-thinning. When $\mathrm{nL}^{3}$ exceeds 115 , Kerekes et al. (1992) demonstrates that fibre-fibre contact 
is unavoidable, which may be the main reason for the shear thinning behaviour. When $\mathrm{nL}^{3}$ is lower than 115 , the magnitude of the increase in relative viscosity is quite small. It is inconvenient if an analysis of fibre length by the viscosity method is conducted at such low concentrations. In the absence of a theory at high concentrations when fibre-fibre contact emerges, it is therefore necessary to resort to an experimental method so that a well-defined correlation between fibre suspension viscosity and fibre length can be determined.

For measuring fibre suspensions' viscosity, two factors should be carefully taken into account when selecting a suitable viscometer: wall effect and wall slip problem. The wall effect refers to the influence of the finite dimensions on the viscosity. In the majority of earlier experimental measurements, attempts were made to avoid wall effect by using a larger ratio of dimensions, such as by Blackeney (1966), Nawab and Mason (1958), and Attanasio (1972). The problem is now solved by Dinh et al. (1984) theoretically and verified experimentally by Bibbo (1987). When the ratio between the characteristic dimension of a viscometer and the length of fibre is greater than 1.2 , the wall effect is not significant since most of the fibre will be aligned along the streamline after a short time (Bibbo 1987).

In most reports on the measurement of fibre suspension viscosity, such as those listed in the reviews by Dinh et al.(1984), Powell et al (1985), Petrie (1999) and Derakhshandeh et al. (2011), the fibre length did not exceed $3 \mathrm{~mm}$. Therefore, commercially available viscometers can be satisfied with the dimension requirement, e.g. concentric cylinders, parallel plates, cone-and-plate, and capillary viscometer. For measuring the viscosity of longer fibre suspensions, a fundamental difficulty is in the particle's dimensions, which are of the same order of magnitude as the flow field's characteristic length in standard viscometers. A bespoke device needs to be designed so that a gap must be kept wide enough to accommodate the fibres.

Another factor to consider is the wall slip effect. Wall slip effect refers to a lower concentration of suspension near the wall when compared to the bulk concentration, because of the displacement of the dispersed phase away from solid boundaries in the flow of two-phase systems (Barnes 1995). Although the wall slip problem may be minimised by using rough surfaces, such as sand paper or serrated plates, this approach still includes the difficulties associated with sample loading (Derakhshandeh et al. 2010a; 2010b). Due to the above limitations, Barnes et al. (1990) proposed the use of a vane-in-cup configuration to avoid the wall slip, which was adopted in this work.

The basic principle for the measurements of viscosity is in the determination of shear stress at an applied shear rate or vice versa. In a vane-in-cup configuration, direct determination of shear rate is 
difficult, particularly at a higher rotation speed for the vane-in-cup viscometer (Barnes and Carnali 1990; Barnes and Nguyen 2001). Although an ultrasonic Doppler velocimetry can be used to measure the velocity profile between the gaps of the rotors and stators (Derakhshandeh et al. $2010 \mathrm{~b})$, the cost of the viscometer will be much increased. To avoid direct determination of shear rate and shear stress and enable a cost efficient implementation, Metzner's method (1957) can be adopted to the vane-in-cup viscometer, which is based on the following two principles. The first is that the effective shear rate in a stirred liquid vessel is linearly related to the rotational speed in a laminar flow. The relationship between the rotation speed of the vane and the shear rate generated in the liquid can thus be determined using a well-defined non-Newtonian liquid. The second principle is that the Reynolds number $(R e)$ and power number $\left(N_{p}\right)$ have a well-defined relationship in a laminar flow. The effective viscosity of an unknown non-Newtonian fluid (such as fibre suspensions) can thus be determined by a pre-determined relationship between $N_{p}$ and $R e$ for Newtonian fluids in the same stirred vessel.

In summary, to assess fibre length by measuring the viscosity of the fibre suspension, the suspension should be in a semi-concentrated regime so that the change in viscosity can be observed clearly. There are no existing formulae used for fibres with a relatively large length in this concentration regime due to their complicated interactions. Currently, this important relationship between the suspension viscosity and fibre length needs to be determined by an experimental method. The goal of this paper is to develop a correlation between the shear viscosity of fibre suspension and fibre length using a vane-in-cup viscometer with bespoke dimensions. The obtained viscosity relation is then validated using a suspension with known fibre length.

\section{Experimental}

\section{Materials}

Two types of carbon fibres were used in the experimental work. One type was Toho Tenax standard modulus T140 chopped carbon fibres with a nominal length of $3 \mathrm{~mm}, 6 \mathrm{~mm}$, and $12 \mathrm{~mm}$. The other type was Toray T800s, which was chopped to $12 \mathrm{~mm}$ long in our laboratory. Detailed properties of the fibres used are listed in Table 1.

Table 1. Properties of the fibres used in the work

\begin{tabular}{lccccc}
\hline Fibre & $\begin{array}{c}\text { Length } \\
(\mathrm{mm})\end{array}$ & $\begin{array}{c}\text { Diameter } \\
(\mu \mathrm{m})\end{array}$ & $\begin{array}{c}\text { Aspect } \\
\text { ratio }(\mathrm{L} / \mathrm{d})\end{array}$ & $\begin{array}{c}\text { Tensile strength } \\
(\mathrm{MPa})^{*}\end{array}$ & $\begin{array}{c}\text { Young's } \\
\text { modulus (GPa)* }\end{array}$ \\
\hline Carbon fibre (T140) & $3.20 \pm 0.12$ & $7.11 \pm 0.06$ & 450.07 & 4275 & 225 \\
\hline Carbon fibre (T140) & $6.03 \pm 0.09$ & $7.05 \pm 0.16$ & 855.32 & 4275 & 225 \\
\hline Carbon fibre (T140) & $12.51 \pm 0.21$ & $7.09 \pm 0.09$ & 1764.46 & 4275 & 225 \\
\hline
\end{tabular}




\begin{tabular}{llllll}
\hline Carbon fibre (T800S) & $11.20 \pm 0.08$ & $5.07 \pm 0.11$ & 2209.07 & 5880 & 294 \\
\hline
\end{tabular}

*Manufacturer's data

Glycerol aqueous solutions with different viscosities were used as Newtonian liquids to obtain the relationship between the Reynold number and the power number of the bespoke viscometer. The water content in glycerol varied from 0 - $15 \%$ (wt), which has a viscosity range from $80 \mathrm{mPa}$.s to $1200 \mathrm{mPa}$.s. The viscosity of the glycerol solutions was measured using Brookfield LVDV-II viscometer. The spindle used was \#2LV-CYL at 3-30 rpm. The solution containing $11 \%(\mathrm{wt})$ of water (viscosity $=150 \mathrm{mPa} . \mathrm{s}$ at $23^{\circ} \mathrm{C}$ ) was used as the suspending liquid for the carbon fibres because of its high dispersing effectiveness.

A 1.0\% (wt) aqueous solution of 2-Hydroxyethylcellulose (HEC) was used to obtain the relationship between shear rate and the rotation speed of the bespoke viscometer's vane. The HEC was Natrosol $250 \mathrm{HHR}$, which has a molecular weight of $1.3 \times 10^{6} \mathrm{~g} / \mathrm{mol}$. The solution was prepared by adding the HEC powder gradually into deionized water while stirring. The stirring was maintained for at least two hours until the solution became clear. After preparation, the solutions were stored for 24 hours before use. The viscosity of the two solutions was measured using the Brookfield viscometer. Powerlaw equations were used to correlate the experimental data, as in Eq. (4) (Bhatia 2011).

$$
\mu=10.9062 \dot{\gamma}^{0.3268-1}
$$

\section{Bespoke viscometer}

A vane-in-cup viscometer was designed and manufactured in-house to measure the viscosity of fibre dispersion. A schematic diagram of the device is shown in Fig. 1. The outer cylinder was made of Perspex $^{\circledR}$ with a diameter of $192 \mathrm{~mm}$ and a height of $250 \mathrm{~mm}$. The rotating vane was made of stainless steel. It had four $3 \mathrm{~mm}$ thick blades with a cross-configuration. The vane had a diameter of $150 \mathrm{~mm}$, and a height of $150 \mathrm{~mm}$.

The vane was mounted on a Silverson ${ }^{\circledR}$ overhead stirrer, the rotation speed of which can be continually adjusted. The outer cylinder was installed on a heavy metal platform via a short shaft fitted inside a low-friction bearing fixed on the platform. The shaft had a diameter of $30 \mathrm{~mm}$ and a length of $30 \mathrm{~mm}$. When the vane rotated, the reaction torque of the outer cylinder was measured as that which had to be applied to restrain it from rotation. The force to resist the rotation was measured using a load cell (Model 1004, Tedea \& Huntleigh) and then transferred to a computer via a Vishay ${ }^{\circledR}$ VT100 data-logger. The measured reaction torque of the outer cylinder was calibrated against a torque transducer installed on the shaft of the vane. The torque transducer was DRFL-1b2Nm supplied by Applied Measurements Ltd (UK). 
On the wall of the outer cylinder a thermocouple was installed $60 \mathrm{~mm}$ from the bottom to measure the temperature change of the liquid during measurement. The temperature was recorded via a Pico ${ }^{\circledR}$ TC-08 data-logger.

The temperature of the suspending liquid was not controlled in the experiment because the following had to be taken into account. Firstly, glycerol has a high viscosity, and the gap between the tip of the vane and the outer cylinder is $21 \mathrm{~mm}$. There are no effective ways of cooling and consequently a temperature gradient in the liquid will appear.. Secondly, the relationship between temperature and glycerol and its aqueous solution is well established. When the temperature is known, the viscosity of the aqueous glycerol solution can be calculated accurately. In this work, Cheng's correlation (2008) for the viscosity and density of glycerol aqueous solution with temperature was adopted to calculate the viscosity based on the recorded temperature.

The relative error of the bespoke viscometer was estimated as follows. The load cell has an accuracy of $1 \mathrm{~g}$. The length of the arm $L$ was $55 \mathrm{~mm}$. The accuracy of the torque measurement is therefore $9.81(\mathrm{~N} / \mathrm{kg}) \times 1 / 1000(\mathrm{~kg}) \times 0.055(\mathrm{~m})=5.4 \times 10^{-4}(\mathrm{Nm})$, and the relative error is $5.4 \times 10^{-4} / 0.074=$ $0.7 \%$ (where $0.074 \mathrm{Nm}$ is the lowest torque measured in our experimental work). When we further shortened the arm, the signal had a larger noise. Therefore, this arm length was chosen with a compromise between accuracy and noise level.

The sensitivity of the bespoke viscometer was estimated as follows. The minimum force to make the outer cylinder rotate was measured as $1.45 \mathrm{~N}$, which corresponded to a torque sensitivity of 0.08 N.m when the arm length is $55 \mathrm{~mm}$. At a lower rotation speed, the load cell was not able to detect any force.

\section{Determination of the power number equation}

In a stirred vessel, the power drawn by the stirrer in terms of the power number $\left(N_{\mathrm{p}}\right)$ is dependent on the Reynolds number (Re), the Froude number $(F r)$ and the geometry of the vessel (Tatterson 1991). The bespoke viscometer is un-baffled to avoid fibre catching on the baffled plates. In an unbaffled stirred vessel Fr should be included in the power number equation (Scargiali et al. 2013). Also, the bespoke viscometer has fixed geometric sizes. The geometry can thus be excluded in the power number equation. Subsequently, the power number equation can be written as Eq. (5) when the bespoke viscometer is regarded as a stirred vessel.

$$
N_{p}=K \operatorname{Re}^{a} \operatorname{Pr}^{b}
$$


where $K$ is a proportional constant. $N_{p}, R e$, and $F r$ are defined in Eqs. (6)-(8). The flow in a vane-incup configuration can be approximated as a Couette flow at low Reynolds numbers (Barnes and Carnali 1990). Therefore, the gap between the tip of the vane and the outer cylinder was used in the definition of the Reynolds number (Lathrop et al. 1992).

$$
\begin{aligned}
& R e=\frac{\rho N D_{i}\left(D_{o}-D_{i}\right)}{2 \mu} \\
& F r=\frac{N^{2} D_{i}}{g} \\
& N_{p}=\frac{P}{\rho N^{3} D_{i}^{5}}
\end{aligned}
$$

where $\rho$ is the density of the suspending liquid, $N$ is the rotation speed $(\mathrm{rev} / \mathrm{s}), D_{0}$ is the diameter of the outer cylinder, $D_{i}$ is the diameter of the vane, $g$ is the gravitational acceleration, $\mu$ is the viscosity of the liquid, and $P$ is the power consumption of the vane, which equals $2 \pi N T$, where $T$ is the reaction torque needed to restrain the rotation of the outer cylinder while the vane is rotating at a speed of $N(\mathrm{rev} / \mathrm{s}) . T=F x$, where $F$ is the force that the load cell measured and $x$ is the moment arm length.

The values of $K, a$ and $b$ in Eq. (5) were determined by multi-linear regression of the values of $N_{p}, R e$ and $F r$, which were obtained by varying the rotation speed of the vane from 1.67 to $3 \mathrm{rev} / \mathrm{s}$ and the viscosities of the glycerol aqueous solution. The Reynolds number was kept lower than 50 to remain within the laminar regime (in Couette flow, the Reynolds number should be lower than 60 for laminar flow). Substitution of the determined values of $K, a$ and $b$ into Eq. (4) gives Eq. (9).

$$
N_{p}=10.36 \operatorname{Re}^{-0.39} \mathrm{Fr}^{0.10}
$$

In Fig. 2, the power number $\left(N_{\mathrm{p}}\right)$ are plotted against the product of Re raised a power of -0.39 and $\mathrm{Fr}$ raised a power of 0.10 . The straight line is the regression line of $N p$ and $\operatorname{Re}^{-0.39} \mathrm{Fr}^{0.10}$. The correlation coefficient $\mathrm{R}^{2}=0.98$; the relative standard deviation of estimate of $N \mathrm{p}$ is $3.3 \%$; the relative standard deviation of the $K$ is $2.0 \%$. The relative standard deviation of the viscosity is mainly caused by the relative standard deviations of $N p$ and the $K$, which is estimated to be $1 / 0.39 \times\left[(2.0 \%)^{2}+(3.3 \%)^{2}\right]^{1 / 2}=$ $9.9 \%$

\section{Determination of the shear rate of the vane}


The shear rate generated by the rotating vane was determined using the well-defined shear-thinning liquid 2-hydroxyethylcellulose aqueous solution. About $3500 \mathrm{~mL}$ of the solution was put into the outer cylinder and started rotating the vane at various speeds. The viscosity of the solution at a rotation speed was calculated using Eq. (9) according to the measured power number at that rotation speed. The shear rate generated by that rotation speed was calculated by substitution of the viscosity value into Eq. (4). The shear rates obtained were plotted against the corresponding rotation speeds. Linear regression was used to get the relationship between the rotation speed of the vane and the shear rate generated in the suspension, as in Eq. $(10)\left(R^{2}=0.99\right)$. The positive intercept on the rotation axis is due to the sensitivity of the torque measurement method.

$$
\dot{\gamma}=49.88 N-54.96
$$

\section{Measurement of the viscosity of fibre suspension}

About $3500 \mathrm{~mL}$ of suspending liquid was first put into the outer cylinder to a height of $120 \mathrm{~mm}$, which was below the top of the vane. Since the length of the carbon fibre was up to $12 \mathrm{~mm}$, we observed that the fibres rested on the top of the vane when the vane was totally immersed in the liquid. The liquid height $H$ and the bottom clearance $C$ of the vane were maintained at $120 \mathrm{~mm}$ and $15 \mathrm{~mm}$ respectively.

A pre-determined amount of the carbon fibre was added to the liquid and the vane was rotated at a speed of $250 \mathrm{rpm}$, at which all fibre bundles could be dispersed uniformly in the suspending liquid in about 5 minutes. The absence of fibre bundles was examined by a hand-sheet prepared by deposition of the fibre suspension on a fine nylon mesh. If fibre bundles were not found in the sheet visually, the dispersion was considered to be free of fibre bundles. Potential sedimentation of fibres in the glycerol solution was estimated from the time $t_{\mathrm{s}}$ that a single fibre takes to settle over a distance equal to its length, $t_{\mathrm{s}}=8 \mu \mathrm{L} /\left(\Delta \rho \mathrm{d}^{2}[\ln (2 \mathrm{r})-0.72]\right)$ (Happel and Brenner 1986). The $t_{\mathrm{s}}$ ranged from $35 \mathrm{~min}$ for the fibre with an aspect ratio of 450 to $210 \mathrm{~min}$ for the fibre with an aspect ratio of 2209, which is much longer than the duration of a measurement. The homogeneity of the suspension was further examined by regularly sampling $5 \mathrm{~mL}$ of suspension from the top to determine the deviation of the local concentration from the bulk concentration, which was less than $2 \%$. The long characteristic settling time and the small deviation of local concentration from bulk concentration indicated the homogeneity of the suspension. A clear phase separation was only noticed overnight.

After the fibre was dispersed in the suspending liquid, the vane was stopped for 5 minutes, and then the vane was started again to ramp from $100 \mathrm{rpm}$ to $320 \mathrm{rpm}$ with step size of $20 \mathrm{rpm}$. The time 
interval at each step was about 2 minutes. The torque was observed to reach a constant value for each shear rate almost instantaneously. Therefore, the measurements were truly representative of steady state viscosity. To avoid fibre breakage, the maximum speed was $320 \mathrm{rpm}$ (Moledina 2013). The long carbon fibre is difficult to disperse. When dispersed in another vessel and then transferred to the viscometer, the homogeneity of the suspension was observed to be changed. Therefore, the suspension was made in situ.

The torque for calculating the viscosity was taken as the average in each rotation speed in the time interval. Eqs. (9) and (10) were used to calculate the viscosity and the shear rate respectively. The relative viscosity $\mu_{\mathrm{f}} / \mu_{\mathrm{s}}$ was calculated as the ratio of the suspension viscosity to the suspending liquid viscosity. As Barnes (2000) has pointed out, the viscosity of suspensions is directly proportional to the viscosity of the suspending phase. The relative viscosity was thus regarded as constant with the temperature in the range of viscous heating effect (about $1.5^{\circ} \mathrm{C}$ ) for one test.

\section{Results and discussion}

\section{Shear-thinning properties of carbon fibre suspensions}

It is shown in literature that the viscosity of fibre suspension depends on fibre concentration $\left(n L^{3}\right)$, aspect ratio $(L / d)$, curvature, and shear rate. In order to establish a viscosity method to evaluate fibre length, the viscosity of carbon fibre suspension was measured as a function of fibre concentration $\left(n L^{3}\right)$, aspect ratio $(L / d)$ and shear rate. Fig. 3 shows the relative viscosity versus shear rate at various concentrations for the carbon fibres with aspect ratios of 450, 855, 1764 and 2209 respectively. The viscosity of the suspensions of carbon fibres with such large aspect ratios has rarely been reported in literature.

When the aspect ratio of the carbon fibre is 450 , the relative viscosity $\left(\mu_{f} / \mu_{s}\right)$ does not depend on shear rate when $n L^{3}$ is $22.7,63.8$ and 104.9. However, $\mu_{f} / \mu_{s}$ shows a clear dependence on shear rate when $n L^{3}$ increased to 142.7 . With an increase in shear rate, the relative viscosity decreases gradually. When the aspect ratio is $855, \mu_{f} / \mu_{s}$ does not depend on shear rate when $n L^{3}$ is 81.9 . When the $n L^{3}$ values are higher, $\mu_{\mathrm{f}} / \mu_{\mathrm{s}}$ becomes dependent on shear rate. For the fibres with aspect ratios of 1821 and 2209, all the suspensions show shear-thinning behaviour. The higher the value of $n L^{3}$, the more dependent is the viscosity on shear rate.

When there are only hydrodynamic interactions between fibres, the viscosity of fibre suspensions is dependent on total strain rather than strain rate, as demonstrated in Dinh and Bibbo's work. When the shear rate increases, the orientation of fibres are modified along the flow streamlines. There will 
appear a stress overshoot at the start-up of shear flow. After a short period of time, the fibre orientation will reach an equilibrium state, and the viscosity will show shear-rate independence.

Shear thinning behaviour of fibre suspensions has been reported by many other researchers, and the shear-thinning increases with increasing fibre aspect ratio and concentration. However, the shearthinning has yet to be satisfactorily explained. Mongruel et al. (1999) suggested that measurements showing shear thinning behaviour are not a true reflection of hydrodynamic stress. Zirnsak et al. (1994) proposed the possible reasons as the formation of fibre aggregates and anisotropic fibre orientation, but the explanation is not supported by any experimental evidence.

With an increase in fibre aspect ratio and concentration, the rigid rod-like assumption for fibres is not valid. The fibres in a suspension tend to form flocs. Schell and Kerekes (1992) proposed an empirical crowding factor $X=2 / 3 \varphi(L / d)^{2}$ to characterise the tendency of the fibres towards flocculation. When $X>60$, i.e. $\mathrm{nL}^{3}>115$, there is a strong possibility of flocculation, although the concentration is still in semi-concentrated regime, i.e. $\mathrm{n}<(\mathrm{L} / \mathrm{d})^{2}$. Based on the crowding factor, the concentrations examined in this work are plotted in Fig. 4. To the left of the critical value of $\mathrm{nL}^{3}$, shear-thinning was not observed, while to the right of the critical value, shear-thinning behaviour was observed. This indicates that shear-thinning is strongly related to the crowding factor, i.e. flocs formation. In the transiently formed flocs, there are fibre-fibre contacts, which contribute to the stress at low shear rate. At high shear rates, these flocs are ruptured, fibre-fibre contact disappears. The contributions to the stress are only from hydrodynamic interactions.

It can also be noticed that there are shoulders in Fig. 3 for the fibre with an aspect ratio of 2209. This may be due to the fibre bending in shear flow. Blakeney (1966) and Joung et al (2002) show experimentally and theoretically that the shear viscosity of a fibre suspension increases when the fibres become curved. A cylindrical fibre is expected to buckle in the flow gradient plane when the dimensionless group bending ratio (BR) is less than 1 (Forgacs and Mason 1959a).

$$
B R=\frac{E\left(\frac{2.48(L / d)}{\left(\ln (L / d)^{0.5}\right.}-1.50\right)}{2 \mu_{s} \dot{\gamma}(L / d)^{4}}
$$

where $\mu_{\mathrm{s}}$ is the viscosity of suspending liquid, $\dot{\gamma}$ is the shear rate, and $E$ is the Young's modulus of the fibre. The bending ratios of the carbon fibres were plotted against shear rate in Fig. 5 . The viscosity shoulder is observed when the bending ratio is lower than 0.0028 , as shown below the thick dash line. It can be seen that only the carbon fibre with an aspect ratio of 2209 at high rates 
has a bending ratio lower than this value. This indicates that the viscosity of fibre suspensions are affected by bending when the bending ratio is lower than 0.002806 .

\section{Effects of concentration and aspect ratio on relative viscosity}

Concentration is an important parameter that influences the viscosity of fibre suspensions. For sphere particle suspensions, volume fraction is the only factor influencing the relative viscosity. However, for fibre suspensions, volume fraction is sometimes meaningless. In literature, three concentration expressions are used: volume fraction, $n L^{3}$ and $n L^{2} d . n L^{3}$ has a clear physical meaning, which represents the number of fibres swept by a fibre when it rotates around a minor axis.

At a fixed shear rate of $28.2 \mathrm{~s}^{-1}$, the relative viscosity $\left(\mu_{\mathrm{f}} / \mu_{\mathrm{s}}\right)$ of the fibre suspensions is plotted against the three concentration expressions respectively in Fig. 6 . Similar results can be obtained at other shear rates. When the concentration is expressed as $n L^{2} d$, the relative viscosities of the suspensions segregate into different groups with different aspect ratios. In each group, the relative viscosity has an approximate linear relationship with $n L^{2} d$. When the volume fraction $\phi$ is used to plot against the relative viscosity, similar behaviour is shown. However, when $n L^{3}$ is used as the concentration expression, the relative viscosity of the fibre suspensions does not segregate according to fibre aspect ratios. All the data fall on the same straight line.

In a simple shear flow, Koch (1995) developed an equation to calculate the value of fibre orientation distribution, which is $0.371 /(L / d)$. If this is the case, the viscosity should scale with $n L^{2} d$ according to Eqs. (1)-(3). Fig. 6 indicates that the viscosity indeed scales with $n L^{2} d$. However, the value of fibre orientation distribution is increased with fibre length rather than decreases. Koch's equation was obtained by correlation of the suspensions with much shorter length than in this work. As fibre orientation distribution becomes broader due to decreased bending ratio, a large number of fibres are oriented at various angles, which results in an increased relative viscosity.

\section{An empirical network formation-disruption model}

The close relationship between shear-thinning and Kerekes's crowding factor as discussed above suggests that there are not only hydrodynamic interactions between fibres but also flocs formed for longer fibres examined in this work. A theory is not yet available for predicting shear viscosity of fibre suspensions when non-hydrodynamic interactions are involved. For the purpose of this research, attempts are made to correlate the viscosity data with fibre concentration and shear rate. 
Due to the indication of flocs formation, we propose an empirical network formation-rupture model to interpret the shear thinning behaviour. Transient networks (flocs) are formed in the fibre suspension due to fibre interaction at a lower shear rate. These flocs are disrupted when subjected to a higher shear rate, resulting in a lower shear viscosity of the fibre suspension at higher shear rate. Once the shear rate is reduced, the disrupted flocs are formed again, resulting in a higher shear viscosity. The rupture of the structural linkages is proportional to shear rate, resulting in shear-rate dependence.

This behaviour of network formation-rupture can be described using the Cross model (Cross 1965) which is suitable for describing the formation and rupture of structural linkages subjected to shear force. After being transposed into the nomenclature in this paper, the flow behaviour of the fibre suspension can be represented using Eq. (12).

$$
\mu_{f}=\frac{\mu_{f, 0}-\mu_{f, \infty}}{1+\alpha \dot{\gamma}^{m}}
$$

where $\mu_{\mathrm{f}, 0}$ is the limiting shear viscosity at zero shear rate, $\mu_{f, \infty}$ is the limiting viscosity at infinite shear rate, and $m$ is the flow behaviour index of the non-Newtonian fluid. Divided by the viscosity of the suspending liquid $\mu_{\mathrm{s}}$ in Eq. (12), yields Eq. (13) for relative viscosity:

$$
\frac{\mu_{f}}{\mu_{s}}=\frac{\left(\mu_{f} / \mu_{s}\right)_{0}-\left(\mu_{f} / \mu_{s}\right)_{\infty}}{1+\alpha \dot{\gamma}^{m}}
$$

Clearly, the effect of shear on the relative viscosity can be expressed by the denominator of Eq. (13) when there are network formations and ruptures due to the shear. Similarly, the effect of fibre orientation item $\left\langle p_{x}^{2} p_{y}^{2}\right\rangle$ in Eq. (1) can also be expressed as $1 /\left(1+\alpha \dot{\gamma}^{m}\right)$. Substitute $1 /\left(1+\alpha \dot{\gamma}^{m}\right)$ into Eq. (1), yielding:

$$
\frac{\mu_{f}}{\mu_{s}}=1+\frac{\mu_{\text {fibre }}}{\mu_{s}\left(1+\alpha \dot{\gamma}^{m}\right)}
$$

Eq. (14) may be used to describe the change of the viscosity of fibre suspension with fibre concentration and shear rate. A non-linear regression method was adopted to obtain the values of $\alpha$ and $m$. Both the dilute limit $\mu_{\text {fibre }}$ in Eq. (2) and the semi-concentrated $\mu_{\text {fibre }}$ in Eq. (3) were tried to see which is better for fitting the data. When the effect of finite aspect ratio $f(\varepsilon)$ was included in both the $\mu_{\text {fibre }}$ for dilute and semi-concentrated regimes, the correlation had a much higher correlation coefficient $\mathrm{R}^{2}$. When $f(\varepsilon)$ was not included, the correlation coefficient was lower. In all four types of correlation, the use of $\mu_{\text {fibre }}$ for dilute limit including the effect of finite aspect ratio $f(\varepsilon)$ 
has the highest correlation coefficient, which is shown in Fig. 7. The values of $\alpha$ and $m$ are 4.67 and 0.66 respectively. The final equation is given in Eq. (15).

$$
\frac{\mu_{f}}{\mu_{s}}=1+\frac{\pi n L^{3}}{6\left(1+4.67 \dot{\gamma}^{0.66}\right) \ln (2 L / d)}\left(\frac{\ln (2 L / d)+0.64}{\ln (2 L / d)-1.5}+\frac{1.659}{\ln ^{3}(2 L / d)}\right)
$$

The semi-concentrated $\mu_{\text {fibre }}$ assumes all the fibres are aligned uniformly in the suspension, but the dilute limit assumes no fibre interactions. When networks (or flocs) are formed in the suspension, the distance between the flocs would be much larger than the fibre-fibre distance when the fibres are initially uniformly distributed in space. Hence, there are no interactions between flocs, and each floc may be assumed as a slender body. This may account for how the dilute limit $\mu_{\text {fibre }}$ is a better fit.

\section{Testing of the model}

The empirical model, Eq. (15), was used to measure the average fibre length of several mixed carbon fibre samples. The samples were prepared by mixing the Toho Tenax T140 fibre with different nominal length. The suspending liquid used was glycerol aqueous solution with a viscosity of 200 $\mathrm{mPas}$, and a shear rate of $28.2 \mathrm{~s}^{-1}$ was used to conduct the measurement. After the relative viscosity was measured, the length (L) was solved from Eq. (15) using a numerical method. A comparison of the measured length and the actual length is presented in Table 2.

As shown in Table 2, the fibre length measured using the viscosity method is closer to the weight averaged length. The results are consistent with the prediction of Eq. (15). From Eq. (15), the relative viscosity of fibre suspension is approximately proportional to $L^{3}$ when the fibre concentration is fixed. Therefore, long fibre will contribute more to the measured suspension viscosity of mixed fibres, and consequently the fibre length derived from the viscosity is biased towards weight averaged length.

Table 2. The mixed Toho Tenex carbon fibre samples with different ratios of various nominal lengths and the measured lengths using the viscosity method based on Eq. (15).

\begin{tabular}{l|l|l|l|l|l}
\hline $\begin{array}{l}\text { Fibre nominal } \\
\text { diameter }(\mathrm{mm})\end{array}$ & Mass $(\mathrm{g})$ & $\phi$ & $\mathrm{L}_{\mathrm{n}}(\mathrm{mm})$ & $\mathrm{L}_{\mathrm{w}}(\mathrm{mm})$ & $\begin{array}{l}\text { Measured } \\
\text { length }(\mathrm{mm})\end{array}$ \\
\hline 12 & 1.0 & 0.000158 & $12.51 \pm 0.21$ & $12.51 \pm 0.21$ & $10.99 \pm 1.10$ \\
\hline $12 / 6$ & $1.0 / 1.0$ & 0.000311 & $8.19 \pm 0.21$ & $9.27 \pm 0.21$ & $8.75 \pm 0.88$ \\
\hline $12 / 6$ & $1.0 / 2.0$ & 0.000466 & $7.32 \pm 0.21$ & $8.19 \pm 0.21$ & $7.70 \pm 0.77$ \\
\hline $12 / 6 / 3$ & $1.0 / 2.0 / 1.0$ & 0.000621 & $5.49 \pm 0.21$ & $6.94 \pm 0.21$ & $7.49 \pm 0.75$ \\
\hline $12 / 6 / 3$ & $1.0 / 2.0 / 5.8$ & 0.000905 & $3.91 \pm 0.21$ & $4.90 \pm 0.21$ & $6.58 \pm 0.66$ \\
\hline
\end{tabular}

\section{Conclusions}


In this study, an attempt was made to develop a viscosity method to measure the length of short carbon fibres with a large aspect ratio, where the carbon fibre has an aspect ratio from $450-2200$. A bespoke vane-in-cup viscometer was designed to measure the viscosity of the carbon fibre's suspension. The viscosity data was obtained by using a mixer type viscometer method by regarding the measuring device as a stirring vessel and therefore avoiding the direct determination of shear rate and shear stress.

In a glycerol aqueous solution, the viscosity of the carbon fibre suspension is dependent on $n L^{3}$ and the shear rate, and demonstrates a shear-thinning characteristic. The shear-thinning behaviour is strongly related to the crowding factor proposed by Kerekes. When the crowding factor is greater than 115 , shear-thinning emerges. Moreover, the influence of fibre bending on the viscosity emerges when the bending ratio is lower than 0.0028 .

A transient network formation-rupture model with no interactions between flocs is proposed to describe the viscosity of the fibre suspension due to the presence of these non-hydrodynamic interactions between the fibres (flocs formation and fibre bending). The model can be used to correlate the relative viscosity with $n L^{3}$ and shear rate. Based on the model, the effective viscosity of a fibre suspension can be predicted when fibre concentration, fibre aspect ratio and the shear rate are known. By measuring the effective viscosity of a fibre suspension with a known mass concentration using the bespoke viscometer at a known shear rate, the length of the fibre can be calculated from Eq. (15). A fast viscosity method may subsequently be established for the quality control of recycled carbon fibres.

\section{Acknowledgements}

The authors gratefully acknowledge the financial support of the Boeing Company under the Boeing / University of Nottingham Strategic Collaboration in Carbon Fibre Recycling.

\section{References}

Attanasio A, Bernini U, Galloppo P, Segre G (1972) Significance of viscosity measurements in macroscopic suspensions of elongated particles. Transactions of Society of Rheology 16: 147-155.

Barnes HA (1995) A review of the slip (wall depletion) of polymer solutions, emulsions and particle suspensions in viscometers: its cause, character, and cure. Journal of Non-Newtonian Fluid Mechanics 56: 221-251.

Barnes HA (2000). A Handbook of Elementary Rheology. University of Wales Press. 
Barnes HA, Carnali JO (1990) The vane-in-cup as a novel rheometergeometery for shear thinning and thixotropic materials. Journal of Rheology 34: 841-866.

Barnes HA, Nguyen QD (2001) Rotating vane rheometry - a review. Journal of Non-Newtonian Fluid Mechanics 98: 1-14.

Batchelor GK (1970) The stress system in a suspension of force-free particles. Journal of Fluid Mechanics 41: 545-570.

Bhatia R (2011). Effect of molecular mass, concentration and temperature on the rheological properties of non-Newtonian aqueous polymer solutions. University of Cincinnati.

Bibbo MA (1987). Rheology of semi-concentrated fiber suspensions. PhD thesis, MIT.

Blakeney W (1966) The viscosity of suspensions of straight, rigid rods. Journal of Colloids and Interface Science 22: 324-330.

Chaouche M, Koch DL (2001) Rheology of non-Brownian rigid fiber suspensions with adhesive contacts. Journal of Rheology 45: 369-382.

Cheng NS (2008) Formula for the viscosity of a glycerol-water mixture. Industrial \& Engineering Chemistry Research 47: 3285-3288.

Cross MM (1965) Rheology of non-newtonian fluids: a new flow equation for pseudoplastic systems. Journal of Colloids Science 20: 417-437.

Derakhshandeh B, Hatzikiriakos SG, Bennington CPJ (2010a) The apparent yield of pulp fiber suspensions Journal of Rheology 54: 1137-1154.

Derakhshandeh B, Hatzikiriakos SG, Bennington CPJ (2010b) Rheology of pulp suspensions using ultrasonic Doppler velocimetry. Rheological Acta 49: 1127-1140.

Derakhshandeh B, Keekes RJ, Hatzikiriakos SG, Bennington CPJ (2011) Rheology of pulp fibre suspensions: A critical review,. Chemical Engineering Science 66: 3460-3470

Dinh SM, Armstrong RC (1984) A rheological equation of state for semiconcentratedfiber suspensions. Journal of Rheology 28: 207-227.

Folgar F, Tuck CL (1984) Orientation behaviour of fibers in concentrated suspensions. Journal of Reinforced Plastics and Composites 3: 98-119.

Forgacs OL, Mason SG (1959a) Particle motions in sheared suspensions. 9. Spin and deformation of threadlikeparticles. Journal of Colloids and Interface Science 14: 457-472.

Forgacs OL, Mason SG (1959b) Particle motions in sheared suspensions. 10. Orbits of flexible threadlike particles," . Journal of Colloids and Interface Science 14: 473-491

Ganani E, Powell RL (1985) Suspensions of rodlike particles: literature review and data correlations. Journal of Composite Materials 19: 194-214

Goto S, Nagazono H (1986) The flow behaviour of fibre suspensions in Newtonian fluids and polymer solutions.II. Capillary flow. Rheological Acta 25: 246-256 
Happel J, Brenner H, 1986. Low Reynolds Number Hydrodynamics. Martinus Nijhoff Publishers, Dordrecht.

Jeffery GB (1922) The motion of ellipsoidal particles immersed in a viscous fluid. Proceedings of the Royal Society London A102: 161-179.

Jiang G, Pickering SJ, Lester EH, Turner TA, Wong KH, Warrior NA (2009) Characterisation of carbon fibres recycled from carbon fibre/epoxy resin composites using supercritical n-propanol. Composites Science and Technology 69: 192-198.

Joung CG, Phan-Thien N, Fan XJ (2002) Viscosityof curved fibers in suspension. Journal of NonNewtonian Fluid Mechanics 102: 1-17.

Kitano T, Kataoka T (1981) The rheology of suspensions of vinylon fibers in polymer liquids. II. suspensions in polymer solutions. Rheological Acta 20: 403-415.

Koch DL (1995) A model for orientational diffusion in fiber suspensions. Physics of Fluids 7: 20862088.

Lathrop DP, Fineberg J, Swinney HL (1992) Turbulent flow between concentric rotating cylinders at large Reynolds number. Physical Review Letters 68: 1515-1519

Mackaplow MB, Shaqfeh ES (1996) A numerical study of the rheological properties of suspensions of rigid, non-Brownian fibres. Journal of Fluid Mechanics 329: 155-186.

Metzner AB, Otto RE (1957) Agitation of non-Newtonian fluids. AIChE Journal 3: 3-10.

Meyer LO, Schulte K, Grove-Nielsen E (2009) CFRP-recycling following a pyrolysis route: process optimization and potentials. Journal of Composite Materials 43: 1121-1132.

Moledina Z (2013). Carbon fibre breakage during dispersion in a viscous liquid. MSc thesis, University of Nottingham.

Mongruel A, Cloitre M (1999) Shear viscosity of suspensions of aligned non-Brownian fibres. Rheological Acta 38: 451-457.

Nawab M, Mason SG (1958) The viscosity of dilute suspensions of thread-like particles Journal of Physical Chemistry 62: 1248-1253.

Petrich MP, Koch DL, Cohen C (2000) An experimental determination of the stress-microstructure relationship in semi-concentrated fiber suspensions. Journal of Non-Newtonian Fluid Mechanics 95: 101-133.

Petrie CJS (1999) The rheology of fibre suspensions. Journal of Non-Newtonian Fluid Mechanics 87: 369-402.

Rosinger ELJ, Woodhams RT, Chaffey CE (1974) Shear Flow Behavior of Complex Asbestos Fibril Dispersions. Transactions of Society of Rheology 18: 453-466.

Scargiali F, Busciglio A, Grisai F, Tamburini A, Micale G, Brucato A (2013) Power consumption in uncovered unbaffled stirred tanks: influence of the viscosity and flow regime. Industrial \& Engineering Chemistry Research 52: 14998-15005. 
Schell CJ, Kerekes RJ (1992) Characterisation of fibre flocculation regimes by a crowding factor. Journal of Pulp and Paper Science 18: J32-J38.

Shaqfeh E, Fredrickson G (1990) The hydrodynamic stress in a suspension of rods. Physics of Fluids 2: 7-24.

Stover CA, Koch DL, Cohen C (1992) Observations of fibre orientation in simple shear flow of semidilute suspensions. Journal of Fluid Mechanics 238: 277-296.

Tatterson GB ( 1991) Fluid Mixing and Gas Dispersion in Agitated Tanks. Lightening Source.

Yip HLH, Pickering SJ, Rudd CD (2002) Characterisation of carbon fibres recycled from scrap composites using fluidised bed process. Plastics Rubber and Composites 31: 278-282.

Zirnsak MA, Hur DU, Boger DV (1994) Normal stresses in fibre suspensions. Journal of NonNewtonian Fluid Mechanics 54: 153-193. 


\section{List of Tables}

Table 1. Properties of the fibres used in the work

Table 2. A comparison between measured and actual fibre length for testing the developed relationship between the viscosity of fibre suspension and fibre length

\section{List of Figures}

Fig. 1. A schematic diagram of the bespoke vane-in cup viscometer, where the outer cylinder rested on a bearing fixed on a heavy metal platform. T represents a temperature data logger, and $F$ represents a load cell transducer.

Fig. 2. Power number $N_{\mathrm{p}}$ versus the product of $\operatorname{Re}^{-0.39 *} \mathrm{Fr}^{0.10}$ for the bespoke vane-in-cup viscometer when it is regarded as a stirred vessel. The Newtonian liquids used for the measurement were glycerol aqueous solutions with viscosities ranging from $80 \mathrm{mPa}$.s to $1200 \mathrm{mPa}$.s. The solid line is the regressed line with $R^{2}=0.98$. Re was from 7 to 50 , and $F r$ was from 0.04 to 0.15 .

Fig. 3. The relative viscosity $\left(\mu_{\mathrm{f}} / \mu_{\mathrm{s}}\right)$ of the carbon fibre suspensions versus shear rate applied by the bespoke vane-in-cup viscometer, where the aspect ratios $(L / d)$ of the carbon fibres are 450,855 , 1764, 2205 respectively. The markers in the legend represent different $n L^{3}$ values of the suspensions.

Fig. 4. The concentration $\mathrm{nL}^{3}$ of the measured suspensions for different carbon fibre aspect ratios. The vertical line is where $\mathrm{nL}^{3}$ is 115 , i.e. when the Kerekes's crowding factor $X$ is 60 . Shear thinning occurred to the right of the vertical line.

Fig. 5. Bending ratio (BR) of carbon fibres calculated using Eq. (11) versus applied shear rate for different aspect ratios of the carbon fibres. The dash line is where $B R=0.0028$. Extra viscosity increase was observed when BR is below the line.

Fig. 6. The relative viscosity of carbon fibre suspensions at a shear rates of $28.2 \mathrm{~s}^{-1}$ versus concentration expression $n L 2 d$, volume fraction $\phi$, and $n L 3$ respectively. The mark in the legend represents different aspect ratio of the carbon fibres.

Fig. 7. Non-linear regression of the viscosities of the carbon fibre suspensions with fibre concentration $\left(n L^{3}\right)$ and shear rate using Eq. (15) as the model. The solid line is the regressed line with a correlation coefficient $\mathrm{R} 2=0.98$. 


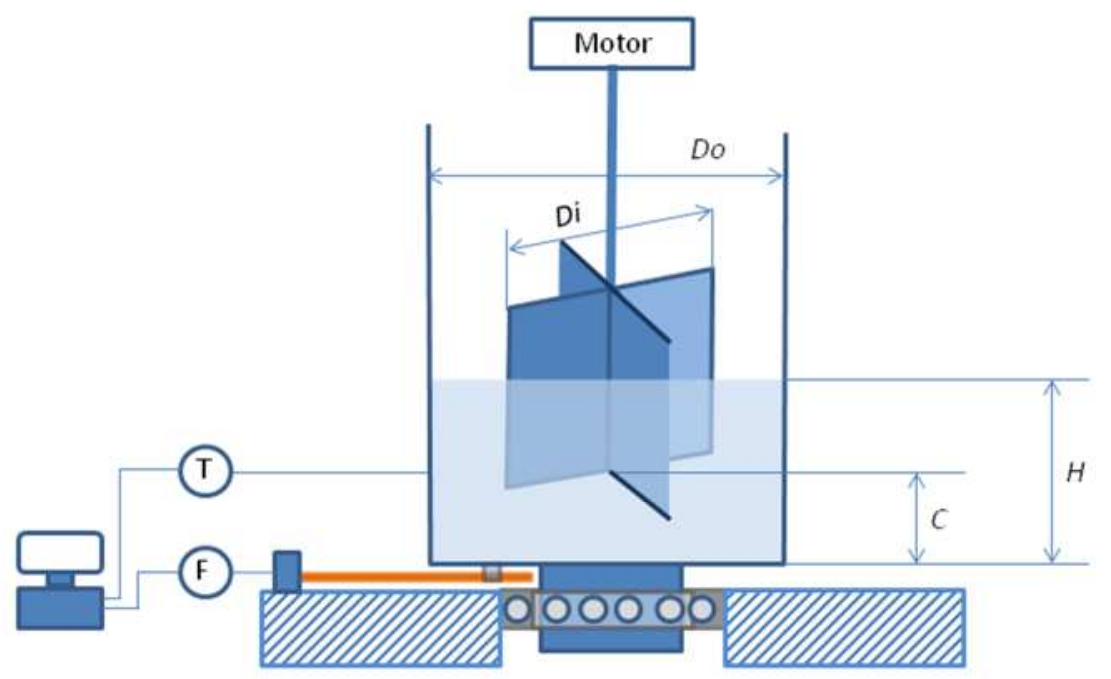

Figure 1

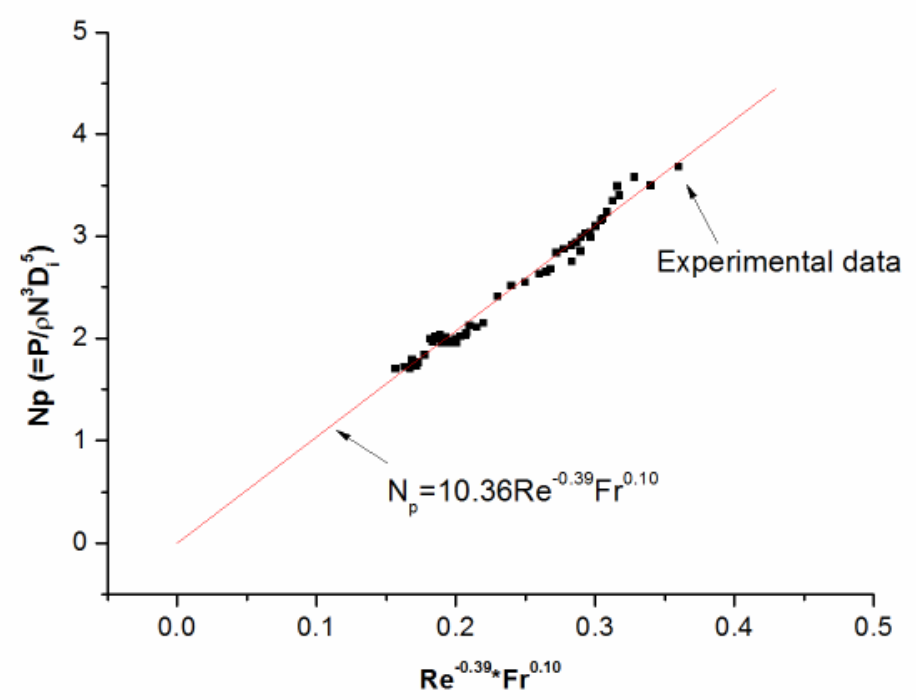

Figure 2 

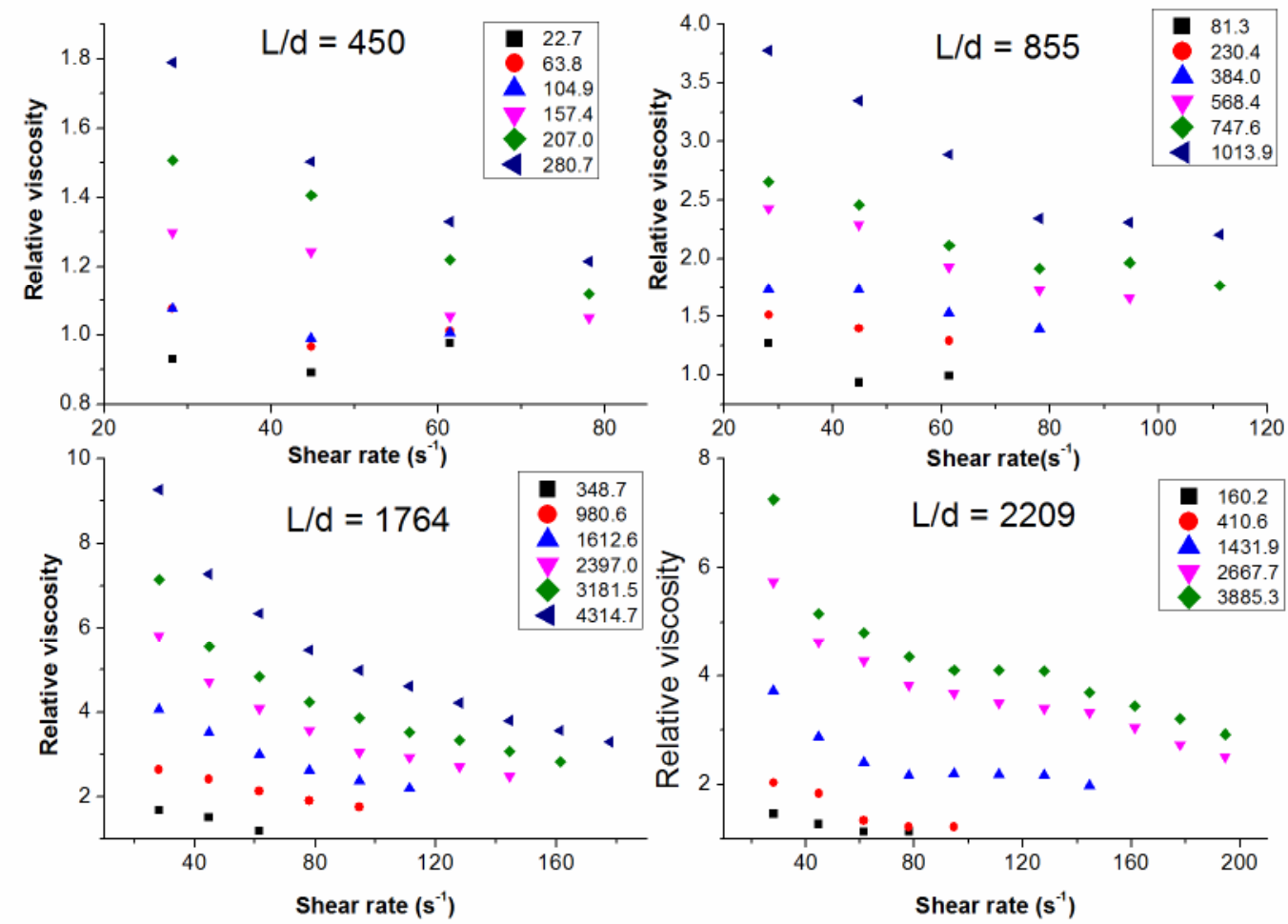

Figure 3

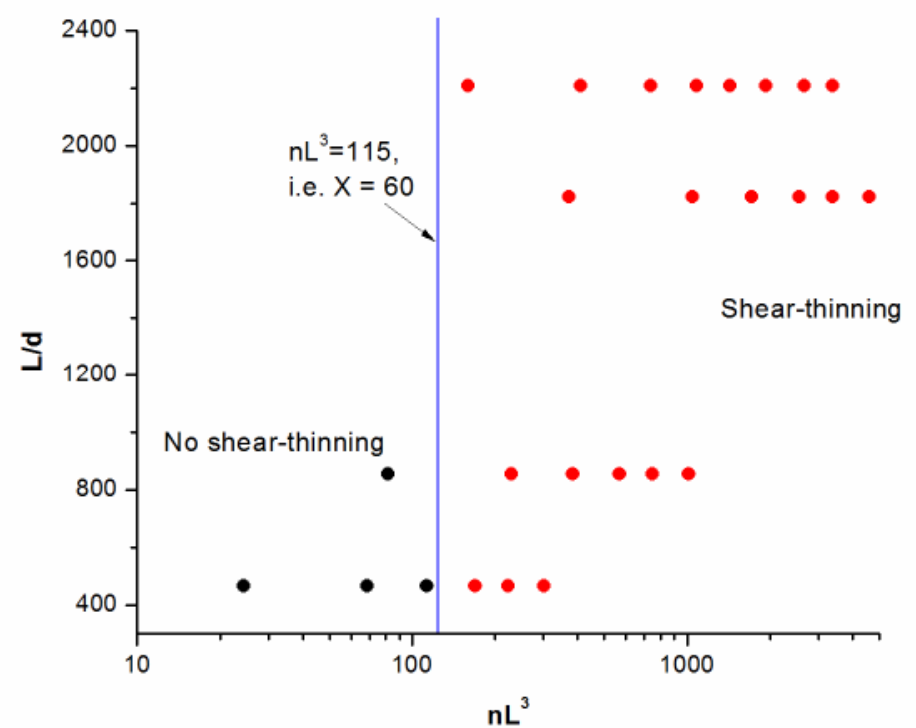

Figure 4 


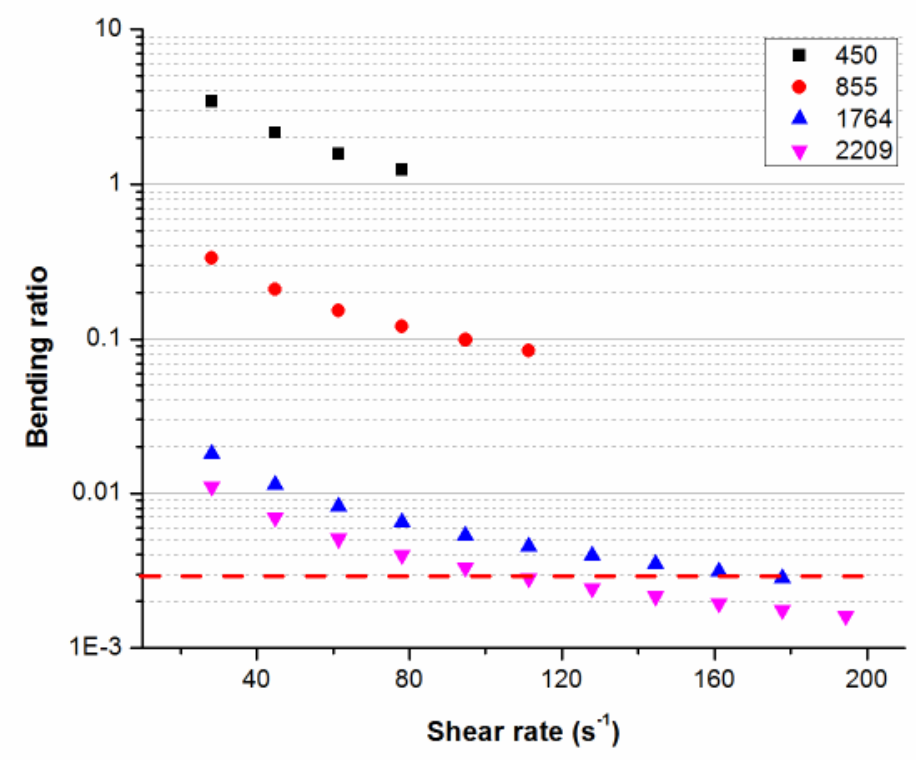

Figure 5

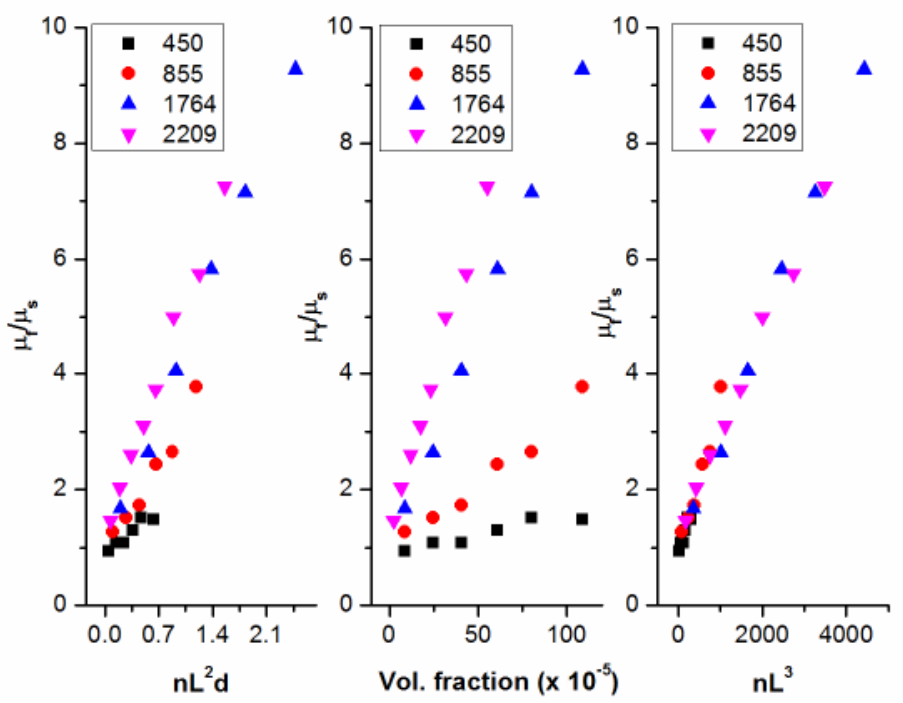

Figure 6 


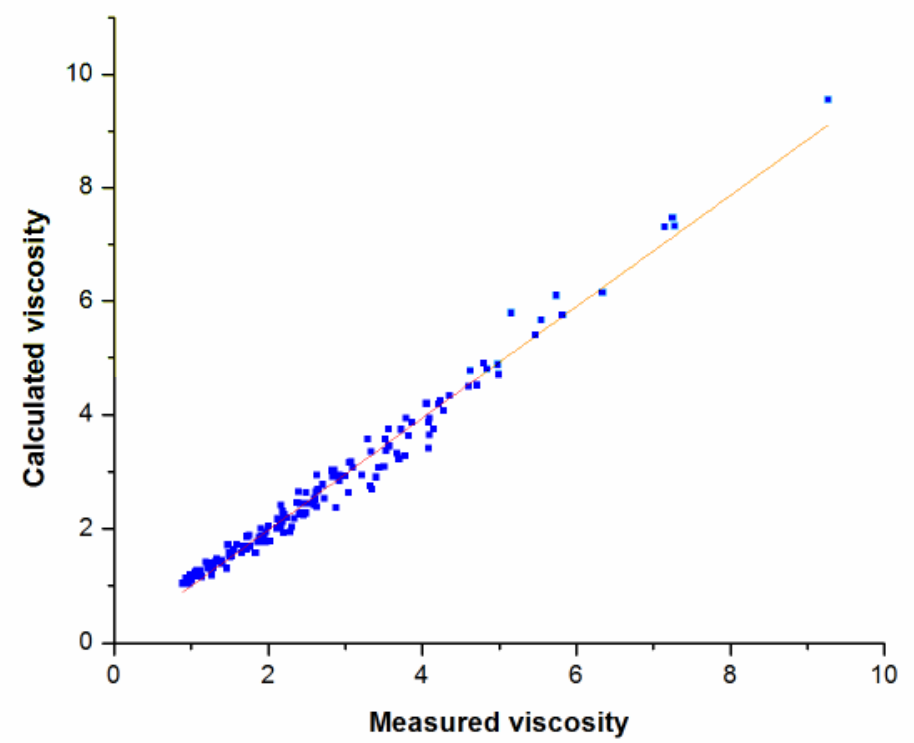

Figure 7 
Table 1. Properties of the fibres used in the work

\begin{tabular}{lccccr}
\hline Fibre & $\begin{array}{c}\text { Length } \\
(\mathrm{mm})\end{array}$ & $\begin{array}{c}\text { Diameter } \\
(\mu \mathrm{m})\end{array}$ & $\begin{array}{c}\text { Aspect ratio } \\
(\mathrm{L} / \mathrm{d})\end{array}$ & $\begin{array}{c}\text { Tensile strength } \\
(\mathrm{MPa})^{*}\end{array}$ & $\begin{array}{r}\text { Young's } \\
(\mathrm{GH}\end{array}$ \\
\hline Carbon fibre (T140) & $3.20 \pm 0.12$ & $7.11 \pm 0.06$ & 450.07 & 4275 & 2 \\
\hline Carbon fibre (T140) & $6.03 \pm 0.09$ & $7.05 \pm 0.16$ & 855.32 & 4275 & 2 \\
\hline Carbon fibre (T140) & $12.51 \pm 0.21$ & $7.09 \pm 0.09$ & 1764.46 & 4275 & 5880 \\
\hline Carbon fibre (T800S) & $11.20 \pm 0.08$ & $5.07 \pm 0.11$ & 2209.07 & 2 \\
\hline
\end{tabular}

*Manufacturer's data

Table 2. The mixed fibre with different average length

\begin{tabular}{l|l|l|l|l|l}
\hline $\begin{array}{l}\text { Fibre nominal } \\
\text { diameter }(\mathrm{mm})\end{array}$ & Mass $(\mathrm{g})$ & $\phi$ & $\mathrm{L}_{\mathrm{n}}(\mathrm{mm})$ & $\mathrm{L}_{\mathrm{w}}(\mathrm{mm})$ & $\begin{array}{l}\text { Measured } \\
\text { length }(\mathrm{mm})\end{array}$ \\
\hline 12 & 1.0 & 0.000158 & $12.51 \pm 0.21$ & $12.51 \pm 0.21$ & $10.99 \pm 1.10$ \\
\hline $12 / 6$ & $1.0 / 1.0$ & 0.000311 & $8.19 \pm 0.21$ & $9.27 \pm 0.21$ & $8.75 \pm 0.88$ \\
\hline $12 / 6$ & $1.0 / 2.0$ & 0.000466 & $7.32 \pm 0.21$ & $8.19 \pm 0.21$ & $7.70 \pm 0.77$ \\
\hline $12 / 6 / 3$ & $1.0 / 2.0 / 1.0$ & 0.000621 & $5.49 \pm 0.21$ & $6.94 \pm 0.21$ & $7.49 \pm 0.75$ \\
\hline $12 / 6 / 3$ & $1.0 / 2.0 / 5.8$ & 0.000905 & $3.91 \pm 0.21$ & $4.90 \pm 0.21$ & $6.58 \pm 0.66$ \\
\hline
\end{tabular}

